\title{
Checklist de Bromeliaceae da região da Volta Grande do Xingu, Pará, Brasil
} Checklist of Bromeliaceae in the region of the Volta Grande do Xingu, Pará, Brasil

\author{
Ana Kelly Koch ${ }^{1,2,4}$, Raquel F. Monteiro ${ }^{3}$ \& Anna Luiza Ilkiu-Borges ${ }^{2}$
}

\begin{abstract}
Resumo
Apresenta-se neste trabalho um inventário das espécies de Bromeliaceae da Volta Grande do Xingu (Pará, Brasil), sendo também fornecida uma chave para identificação das espécies da região, e seus respectivos status de conservação quando existentes. O material utilizado foi obtido através das atividades do Projeto Salvamento e Aproveitamento Científico da Flora da Usina Hidrelétrica de Belo Monte, bem como na coleção do herbário MG. Na área de estudo, Bromeliaceae encontra-se representada por 20 espécies e sete gêneros. Tillandsia e Aechmea foram os mais representativos.
\end{abstract}

Palavras-chave: Amazônia brasileira, Belo Monte, Poales.

\begin{abstract}
In this paper is showed a checklist of the species of Bromeliaceae of the Volta Grande do Xingu (Pará, Brazil), where is provided a identification key for the region species, and your respective status of conservation when available. The material used in this study was obtained through the activities of the Project Rescue and Scientific Utilization of the Flora of the Belo Monte hydroelectric dam, executed by Norte Energia S.A and in the collection of herbarium MG. In the study area, Bromeliaceae is represented by 20 species and seven genera. Tillandsia and Aechmea were the most representative.
\end{abstract}

Key words: Brazilian Amazon, Belo Monte, Poales.

\section{Introdução}

Bromeliaceae é uma família essencialmente neotropical composta por 58 gêneros e aproximadamente 3.199 espécies (Luther 2012; World Checklist 2014) herbáceas de hábitos variados e, segundo análises filogenéticas baseadas em dados moleculares, é monofilética (Givnish et al. 2004, 2007, 2011). Atualmente, encontra-se representada por 44 gêneros e 1.323 espécies no Brasil, das quais 1.155 são endêmicas, ocorrendo em todos os ecossistemas desde o nível do mar até elevadas altitudes (Forzza et al. 2014).

Para a Amazônia brasileira foram registradas 132 espécies de Bromeliaceae em 22 gêneros, números baixos quando comparados aos da Mata Atlântica (891 espécies e 30 gêneros), um dos centros de diversidade da família (Forzza et al. 2014; Martinelli et al. 2008). Na Amazônia, as espécies são encontradas com maior frequência em áreas de baixios, campinas, campinaranas e igapós, como apontado por Nogueira-Braga (1977), Ribeiro et al. (1999) e Sousa \& Wanderley (2007). Das 132 espécies registradas para a Amazônia brasileira, 53 também foram registradas no estado do Pará (Forzza et al. 2014).

Inventários ou listagens de espécies de Bromeliaceae nessa região são pouco comuns, destacando-se Dubs (1998) com a flora da família para o Mato Grosso e Ribeiro et al. (1999) com o tratamento taxonômico da família para a Reserva Adolpho Ducke em Manaus. No estado do Pará, os estudos se focaram apenas nas espécies de hábito epifítico, a saber: Koch et al. (2013), realizaram estudo florístico taxonômico das Bromeliaceae epifíticas da Floresta Nacional de Caxiuanã (cinco espécies); Quaresma \& Medeiros (2009) inventariaram a família na APA Ilha do Combu (seis espécies); e Quaresma \& Jardim (2012;

\footnotetext{
'Instituto de Botânica, Núcleo de Pesquisa Orquidário do Estado, Av. Miguel Stéfano 3687, Água Funda, São Paulo, SP, Brasil.

${ }^{2}$ Museu Paraense Emílio Goeldi, Coordenação de Botânica, Av. Perimetral 1901, Terra Firme, Belém, PA, Brasil

${ }^{3}$ Instituto de Pesquisa Jardim Botânico do Rio de Janeiro, R. Pacheco Leão 915, Jardim Botânico, Rio de Janeiro, RJ, Brasil.

${ }^{4}$ Autor para correspondência: anakbio@gmail.com
} 
2013), estudaram a diversidade, a fitossociologia e a distribuição espacial das bromélias epifíticas encontradas por Quaresma \& Medeiros (2009) em ambiente de várzea.

Vê-se que os estudos sobre Bromeliaceae no Pará são pontuais, revelando que ainda há necessidade de conhecer a flora local de diversas áreas do estado, o que provavelmente contribuirá para o aumento de táxons registrados, podendo gerar futuras ações de conservação das espécies de bromélias no Pará e consequentemente na Amazônia.

Dentre as áreas do Pará que necessitam de maior atenção quanto ao conhecimento de sua flora, encontra-se a região da Volta Grande do Xingu. Segundo Salomão et al. (2007), essa região passou por considerável mudança na sua cobertura vegetal original, devido à forte ação antrópica presente na zona de influência da rodovia Transamazônica (BR-230) e suas transversais, o que provocou uma grande e desordenada ocupação humana como resultado de projetos de colonização agrária durante os últimos 30 anos e, atualmente, abriga a construção da Usina Hidrelétrica de Belo Monte.

Embora essa região tenha sofrido por muito tempo a perda considerável da sua cobertura vegetal original, ainda detém diferentes unidades de paisagens, como florestas ombrófilas ou úmidas, vegetação aluvial e florestas secundárias recentes e antigas (MPEG 2008). Entretanto, estas paisagens ainda são pouco conhecidas no que se refere a sua riqueza e composição. Para tanto, as únicas fontes de dados sobre a flora da região da Volta Grande do Xingu, são a caracterização das florestas de Belo Monte (Salomão et al. 2007), o Relatório Final de Vegetação (MPEG 2008) para a implantação da UHE Belo Monte, no qual apenas sete espécies de Bromeliaceae foram citadas e, Mello et al. (2012), que oferece um guia fotográfico da família para a localidade, composto por 15 espécies.

Diante da necessidade de incrementar o conhecimento atual da flora de Bromeliaceae na Amazônia brasileira e no Pará, objetiva-se aqui a apresentação de um checklist das espécies na Volta Grande do Xingu (Pará, Brasil), bem como divulgar uma chave de identificação para as espécies da região e apontar seus respectivos status de conservação.

\section{Material \& Métodos}

A região da Volta Grande do Xingu abrange três municípios: Altamira, Anapu e Vitória do Xingu (Fig. 1), das mesorregiões do baixo e médio
Xingu, no estado do Pará (MPEG 2008). O clima da região é do tipo Am segundo classificação de Köppen, com temperatura média de $26^{\circ} \mathrm{C}$, precipitação anual de $2.289 \mathrm{~mm}$ e umidade relativa entre 78 e $88 \%$ (Sousa Jr. et al. 2006).

A cobertura vegetal é composta por quatro fitofisionomias principais, a saber: floresta ombrófila densa, floresta ombrófila aluvial, floresta ombrófila aberta com palmeiras e floresta ombrófila aberta com palmeiras e cipós, conforme descreve Salomão et al. (2007), além de capoeiras e áreas de pastagens.

O material utilizado neste trabalho foi obtido através das atividades do Projeto Salvamento e Aproveitamento Científico da Flora da Usina Hidrelétrica de Belo Monte, como parte do Plano Básico Ambiental executado pela Norte Energia S.A., na área de instalação da UHE $\left(03^{\circ} 22^{\prime} 00^{\prime}\right.$ 'S/ $\left.51^{\circ} 56^{\prime} 00^{\prime} \mathrm{W}\right)$, nos municípios de Vitória do Xingu e de Altamira. As amostras botânicas foram coletadas entre junho de 2011 e dezembro de 2013 pelas equipes de salvamento do projeto, abrangendo todas as unidades de paisagens reconhecidas por Salomão et al. (2007). Além disso, foram consultadas as coleções dos herbários do Museu Paraense Emílio Goeldi (MG), Embrapa Amazônia Oriental (IAN), Jardim Botânico do Rio de Janeiro (RB) e o Relatório Final de Vegetação (MPEG 2008).

Os espécimes resgatados passaram por triagem e posterior destinação (exsicatas, cultivo

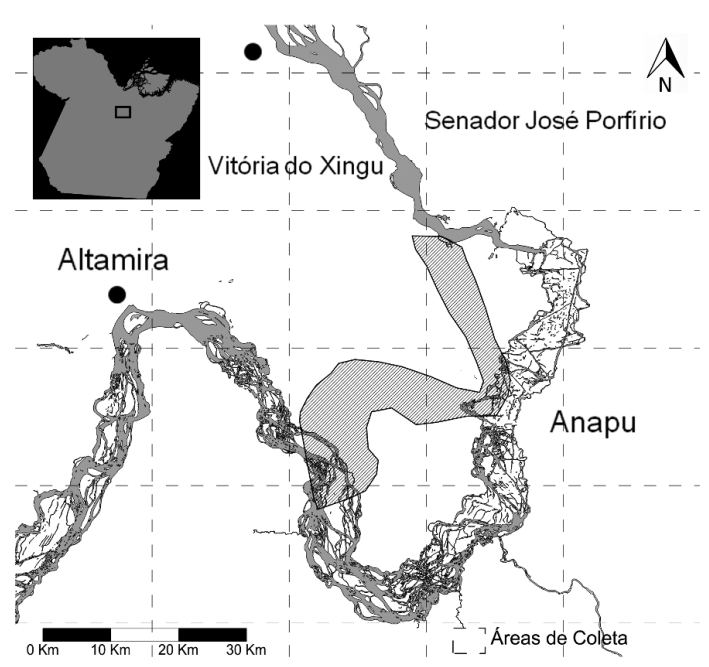

Figura 1 - Localização da Volta Grande do rio Xingu, Pará, Brasil.

Figure 1 - Location of the Volta Grande do rio Xingu, Pará, Brazil. 
Tabela 1 - Lista dos táxons registrados na região da Volta Grande do Xingu. EP: epífita; TE: terrícolas.*Material adicional examinado.

Table 1 - List of taxa recorded in the region of the Volta Grande do Xingu. EP: epiphytes; TE: terrestrial.*Additional material examined.

\begin{tabular}{|c|c|c|}
\hline Táxon & Voucher & Hábito \\
\hline Aechmea bromeliifolia (Rudge) Baker & Dias, A. et al. 601 (MG) & EP \\
\hline Aechmea castelnavii Baker & Gomes, D. PSACF $648(\mathrm{MG})$ & EP \\
\hline Aechmea mertensii (G.Mey.) Schult. \& Schult.f. & Raul, F. PSACF $300(\mathrm{MG})$ & EP \\
\hline Aechmea setigera Mart. ex Schult. \& Schult.f. & imagem (Mello et al. 2012) & EP \\
\hline Aechmea tocantina Baker & Raul, F. PSACF 389 (MG) & EP \\
\hline Ananas ananassoides (Baker) L.B.Sm. & imagem (Mello et al. 2012) & $\mathrm{TE}$ \\
\hline Ananas nanus (L.B.Smith) L.B.Smith & Salomão, R. 981 (MG) & $\mathrm{TE}$ \\
\hline Araeococcus micranthus Brongn. & Cavalcante, P. \& Silva, M. 2854 (MG) & EP \\
\hline Bromelia grandiflora $\mathrm{Mez}$ & imagem (Antonio, L.C.) & $\mathrm{TE}$ \\
\hline Bromelia goeldiana L.B.Sm. & imagem (Mello et al. 2012) & $\mathrm{TE}$ \\
\hline Guzmania lingulata (L.) Mez & Barbacena, V. PSACF 140 (MG) & EP \\
\hline Tillandsia adpressiflora Mez* & Egler, W. $959(\mathrm{MG})$ & EP \\
\hline Tillandsia anceps Lodd & Vasconcelos, R. et al. 25 (MG) & EP \\
\hline Tillandsia bulbosa Hook.f. & Abreu, J. PSACF 137 (MG) & $\mathrm{EP}$ \\
\hline Tillandsia fasciculata $\mathrm{Sw} . *$ & Rabelo, B. $1243(\mathrm{MG})$ & EP \\
\hline Tillandsia monadelpha (E.Morren) Baker* & Costa Neto, S. et al. $130(\mathrm{MG})$ & EP \\
\hline Tillandsia paraensis $\mathrm{Mez}$ & Dias, A. et al. $529(\mathrm{MG})$ & EP \\
\hline Tillandsia streptocarpa Baker & Souza, S. et al. 1 (MG) & EP \\
\hline Tillandsia tenuifolia $\mathrm{L} . *$ & Paula, J. \& Mendonça, R.C. 1235 (MG) & EP \\
\hline Werauhia gigantea (Mart. ex Schult. \& Schult.f.) J.R. Grant* & Pires, J. \& Silva, N. 11722 (IAN) & EP \\
\hline
\end{tabular}

e/ou reintrodução) conforme necessário. Amostras férteis foram herborizadas de acordo com Fidalgo \& Bononi (1984) e depositadas no herbário MG. Amostras estéreis foram coletadas e cultivadas na casa de vegetação do Centro de Estudos Ambientais (CEA) da Norte Energia S.A. para florescimento e posterior herborização. Para as espécies que que foram registradas apenas por fotografias, são citadas na Tabela 1 as devidas referências e autorias das imagens utilizadas no presente estudo e/ou ainda amostras do herbário MG utilizadas como material de referência.

A identificação das espécies foi baseada em bibliografia específica Mez (1891), Smith \& Downs (1974, 1977 e 1979) e, quando necessário, comparação com amostras de herbários. Para as abreviações dos nomes dos autores das espécies utilizou-se Brummitt \& Powell (1992). Os dados de distribuição geográfica das espécies estão de acordo com Forzza et al. (2014). Também foi confirmada a ocorrência de espécies em listas de espécies ameaçadas e a classificação das mesmas em diferentes categorias de grau de ameaça consultando Martinelli et al. (2013), Forzza et al. (2013), IBAMA (2014) SEMA-PA (2014) e IUCN (2014).

A construção da chave de identificação das espécies foi baseada na análise de espécimes herborizados e em espécimes vivos cultivados no CEA quando necessário. Além disso, foram obtidos dados na literatura supracitada. A terminologia morfológica está de acordo com Scharf \& Gouda (2008).

\section{Resultados e Discussão}

Na região da Volta Grande do Xingu, Bromeliaceae encontra-se representada por 20 espécies distribuídas em sete gêneros, correspondendo a cerca de $38 \%$ das espécies registradas para o Pará (53 espécies), sendo 16 epífitas e quatro terrícolas (Tab. 1). Os gêneros Tillandsia e Aechmea foram os mais representativos com sete e seis espécies respectivamente, seguidos por Bromelia e Annanas 
com duas espécies e Araeococcus, Guzmania e Werauhia, com uma espécie cada.

Quando comparado com os trabalhos que trataram a família para o Pará, o presente estudo mostra uma maior biodiversidade, apresentando mais que o dobro das espécies reportadas por Quaresma \& Medeiros (2009) e Koch et al. (2013), que registraram seis e cinco espécies, respectivamente. Mesmo considerando somente as espécies epífitas da Volta Grande do Xingu, o resultado ainda é bastante expressivo em relação a riqueza reportada para as outras áreas do Estado.

Em relação à exclusividade das espécies quando comparada com os demais estudos sobre Bromeliaceae no Pará, 12 são exclusivas da Volta Grande do Xingu: Aechmea castelnavii, A. tocantina, Ananas ananassoides, A. nanus, Bromelia grandiflora, B. goeldiana, Tillandsia adpressiflora, T. fasciculata, T. monadelpha, T. paraensis, T. streptocarpa e T. tenuifolia. Na APA Ilha do Combu (Quaresma \& Medeiros 2009) e, na Floresta Nacional de Caxiuanã (Koch et al. 2013) não houve exclusividade de espécies.

Entre as espécies inventariadas, Aechmea mertensii, Guzmania lingulata e Tillandsia bulbosa são comuns entre as três áreas comparadas, fato presumido pela boa representatividade destas nos herbários amazônicos, corroborado pela ampla distribuição das mesmas na Amazônia.

Em MPEG (2008) foram reportadas as seguintes espécies: Aechmea mertensii, A. setigera, Ananas nanus, Bromelia goeldiana, Tillandsia bulbosa, T. streptocarpa e Werauhia gigantea. Além dessas Mello et al. (2012) registraram mais dez para a região, sendo Tillandsia tenuifolia registrada pela primeira vez para o Pará, ampliando sua distribuição no Brasil.

Quanto à distribuição das espécies reportadas para a Volta Grande do Xingu, três ocorrem em mais da metade dos Estados brasileiros, a saber: Ananas anassoides (22 estados), Aechmea bromeliifolia (20 estados) e Tillandsia tenuifolia (16 estados) (Forzza et al. 2014). Por outro lado, outras três espécies apresentaram distribuição mais restrita, como Tillandsia fasciculata que ocorre apenas nos estados do Amapá, Amazonas e Pará, Bromelia goeldiana no Amazonas e Pará e Werauhia gigantea no Mato Grosso e Pará (Forzza et al. 2014). Na área de estudo, Aechmea castelnavii, A. mertensii, A. tocantina, Bromelia goeldiana, Guzmania lingulata, Tillandsia bulbosa, T. adpressiflora, T. paraensis e T. streptocarpa foram as mais comuns durante as atividades do resgate de flora. Destas, as espécies do gênero Tillandsia foram frequentemente encontradas em floresta ombrófila aluvial, nas margens ou ilhas do rio Xingu. As espécies dos gêneros Aechmea e Bromelia foram encontradas em áreas de floresta ombrófila densa e floresta ombrófila abertas ou ainda, em áreas de vegetação secundária. Guzmania lingulata teve populações encontradas tanto em floresta ombrófila aluvial, quanto em floresta ombrófila densa. Essa última espécie é típica de subosque ou margens de rios com formações florestais mais fechadas, sempre formando pequenas populações, assim como constatado por Koch et al. (2013) na FLONA de Caxiuanã.

As espécies pouco frequentes na região da Volta Grande do Xingu são Tillandsia anceps, encontrada na coleção do herbário $\mathrm{MG}$ e que não havia sido citada nos trabalhos apresentados para a região, e $T$. monadelpha e $T$. tenuifolia, que foram encontradas apenas uma vez e em floresta ombrófila aluvial, entretanto, representadas por muitos indivíduos.

Considerando o status de conservação das espécies encontradas na região de estudo, nenhuma espécie se encontra sob categoria de ameaça ou incluída no Livro Vermelho da Flora do Brasil (Forzza et al. 2013). Todavia, esse estudo e novos esforços que visem ampliar o conhecimento sobre Bromeliaceae no domínio Amazônia poderão subsidiar as próximas avaliações sobre espécies ameaçadas.

Com este checklist foi possível confirmar uma maior diversidade da família Bromeliaceae em determinadas áreas do domínio Amazônia. Neste contexto, na região da Volta Grande do Xingu, pelo menos quatro fitofisionomias diferentes foram intensamente coletadas fato que também pode propiciar uma maior riqueza, enquanto que na APA Ilha do Combu e na FLONA de Caxiuanã, com vegetação predominante de floresta de várzea na primeira e de terra firme na segunda, foram reportadas menos que dez espécies de Bromeliaceae em cada (Quaresma \& Medeiros 2009; Koch et al. 2013). Este estudo demonstra também a importância da continuidade dos trabalhos de flora no Brasil, principalmente na Amazônia brasileira, pois com o mesmo foi possível ampliar a área de ocorrência da maioria das espécies no estado do Pará.

A seguir, é apresentada uma chave para identificação das espécies de Bromeliaceae da região da Volta Grande do Xingu. 


\section{Chave para identificação das Bromeliaceae da região da Volta Grande do Xingu, Pará, Brasil}

1. Plantas terrícolas

2. Inflorescência em espiga estrobiliforme, coma apical presente

3. Coma apical 2 a 3 vezes maior que a inflorescência

Ananas nanus

3'. Coma apical igual ou pouco maior que a inflorescência (nunca até 2 vezes maior)...

2'. Inflorescência nunca estrobiliforme, coma apical ausente

4. Pedúnculo conspícuo, até $30 \mathrm{~cm}$ alt., pétalas roxas Ananas ananassoides (Fig. 3a)

4'. Pedúnculo inconspícuo, imerso no centro da roseta, pétalas magenta....

1'. Plantas epífitas

Bromelia grandiflora (Fig. 3e)

5. Apêndices petalíneos presentes

6. Lâminas foliares de margem inteira Werauhia gigantea

6'. Lâminas foliares de margem espinescente

7. Pedúnculo branco-lanoso; inflorescência em espiga estrobiliforme

7'. Pedúnculo lepidoto; inflorescência em panícula ou fascículos Aechmea bromeliifolia (Fig. 2a)

8. Folhas até $2 \mathrm{~m}$ compr.; brácteas pedunculares com margem inteira Aechmea tocantina (Fig. 2h-i)

8'. Folhas até $1 \mathrm{~m}$ compr.; brácteas pedunculares com margem espinescente

9. Inflorescência recurvada; bráctea floral primária espiniforme Aechmea setigera (Fig. 2f-g)

9'. Inflorescência ereta; bráctea floral primária nunca espiniforme

10. Cálice verde-azulado em direção ao ápice; corola rósea. Aechmea castelnavii (Fig. 2b-c)

10’. Cálice totalmente verde; corola amarela Aechmea mertensii (Fig. 2d-e)

5'. Apêndices petalíneos ausentes

11. Rosetas não acumuladoras de água

12. Frutos do tipo baga e sementes sem apêndices .

Araeococcus micranthus (Fig. 3b)

12'. Frutos do tipo cápsula e sementes com apêndices plumosos

13. Lâminas foliares velutino-cinéreas. Tillandsia streptocarpa (Fig. 4i)

13'. Lâminas foliares nunca velutino-cinéreas

14. Bainhas foliares infladas formando um aspecto bulboso; pétalas arroxeadas Tillandsia bulbosa (Fig. 3i-4a)

14'. Bainhas foliares nunca infladas; pétalas róseas ou brancas

15. Inflorescência complanada com flores dísticas

16. Brácteas florais verdes; pétalas brancas Tillandsia monadelpha (Fig. 4d-e)

16’. Brácteas florais róseas; pétalas arroxeadas Tillandsia anceps

15'. Inflorescência nunca complanada com flores polísticas

17. Flores com pétalas róseas; estames excertos Tillandsia paraensis (Fig. 4f-g)

17'. Flores com pétalas brancas; estames inclusos Tillandsia tenuifolia (Fig. 4h)

11'. Rosetas acumuladoras de água

18. Folhas até $5 \mathrm{~cm}$ larg.; bainhas foliares internamente verdes Tillandsia fasciculata (Fig. 4b-c)

18'. Folhas 10 a $15 \mathrm{~cm}$ larg.; bainhas foliares internamente roxo-vináceas. Tillandsia adpressiflora (Fig. 3g-h) 

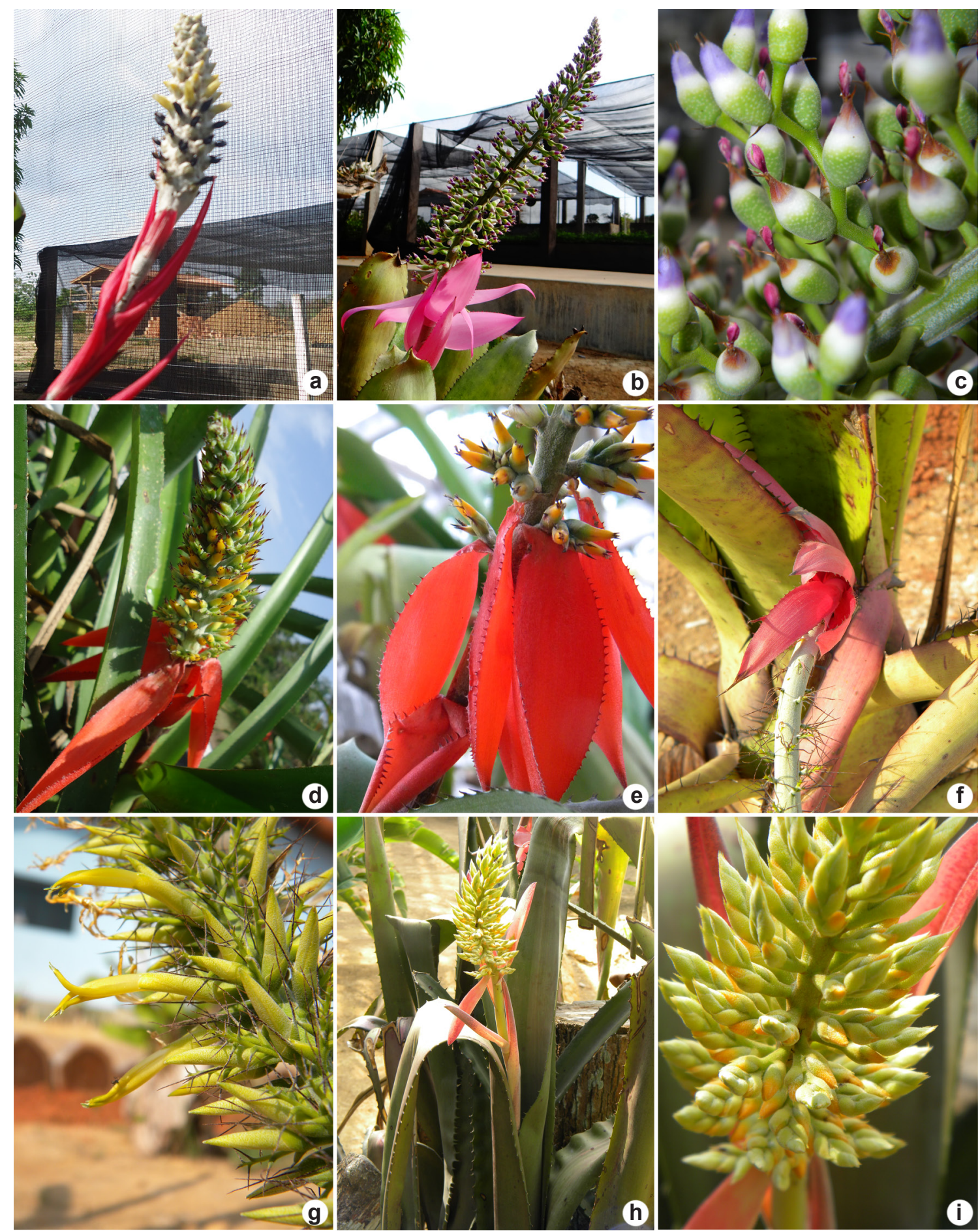

Figura 2 - a. Aechmea bromeliifolia - detalhe da inflorescência; b-c. Aechmea castelnavii - b. detalhe da inflorescência; c. flores e frutos imaturos; d-e. Aechmea mertensii - d. detalhe da inflorescência; e. detalhe das bráteas; f-g. Aechmea setigera $-\mathrm{f}$. detalhe das brácteas da base do pedúnculo; g. detalhe da inflorescência; h-i. Aechmea tocantina - h. hábito fértil; i. detalhe da inflorescência.

Figure 2 - a. Aechmea bromeliifolia - detail of inflorescence; b-c. Aechmea castelnavii - b. detail of inflorescence; c. flowers and imature fruits; d-e. Aechmea mertensii - d. detail of inflorescence; e. detail of bracts; $\mathrm{f}$-g. Aechmea setigera $-\mathrm{f}$. detail of bracts at peduncle base; g. detail of inflorescence; h-i. Aechmea tocantina - fertile habit; i. detail of inflorescence. 

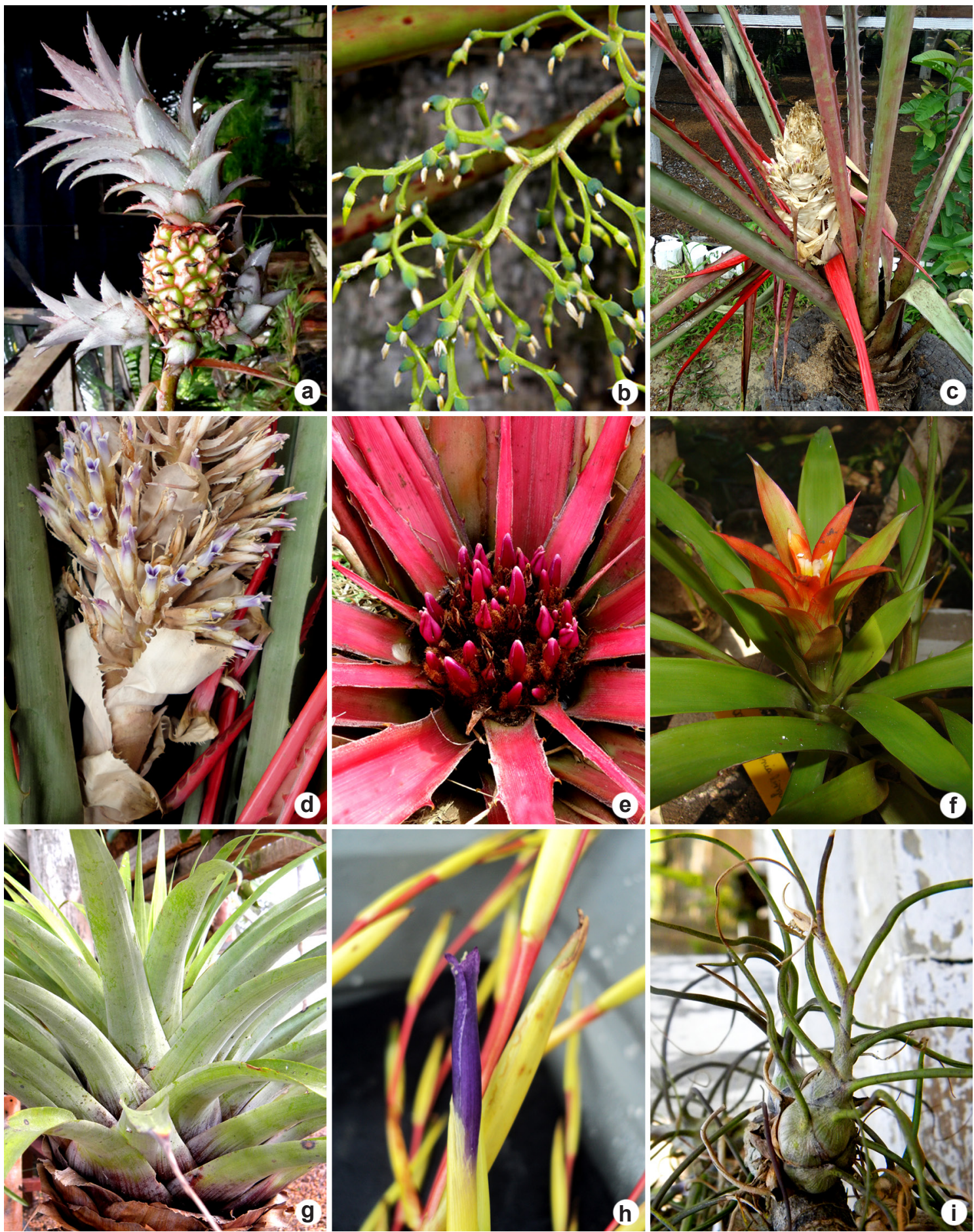

Figura 3 - a. Ananas ananassoides - detalhe da infrutescência; b. Araeococcus micranthus - detalhe da inflorescência; c-d. Bromelia goeldiana - c. hábito fértil; d. detalhe da inflorescência e flores; e. Bromelia grandiflora - detalhe da inflorescência; f. Guzmania lingulata - hábito fértil; g-h. Tillandsia adpressiflora - g. hábito vegetativo; h. detalhe da flor; i. Tillandsia bulbosa -hábito vegetativo.

Figure 3 - a. Ananas ananassoides - detail of infrutescence; b. Araeococcus micranthus - detail of inflorescence; c-d. Bromelia goeldiana - c. fertile habit; d. detail of inflorescence and flowers; e. Bromelia grandiflora - detail of inflorescence; f. Guzmania lingulata - fertile habit; g-h. Tillandsia adpressiflora - g. vegetative habit; h. detail of flower; i. Tillandsia bulbosa - vegetative habit. 

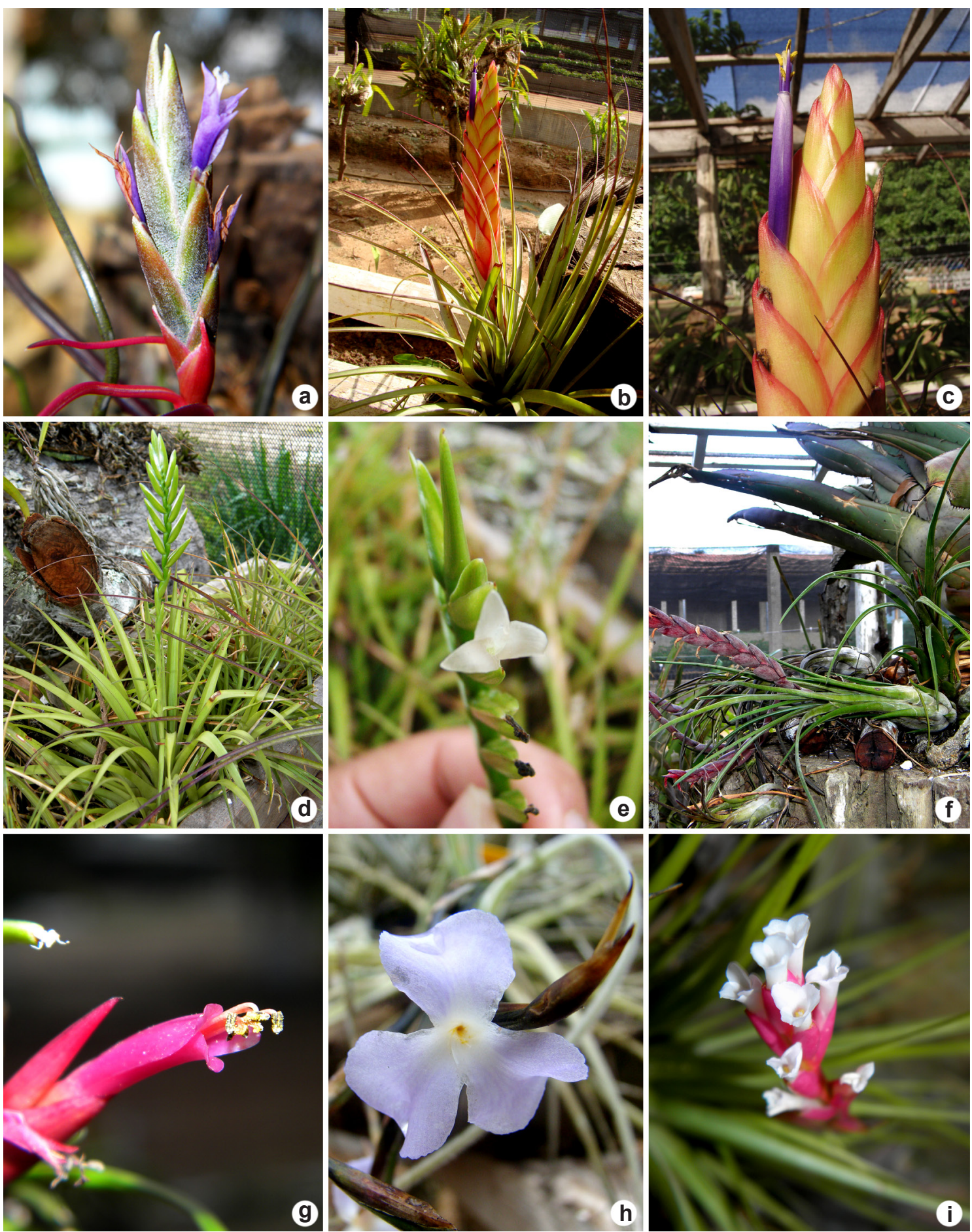

Figura 4 - a. Tillandsia bulbosa - detalhe da inflorescência; b-c. Tillandsia fasciculata - b. hábito fértil; c. flor; d-e. Tillandsia monadelpha - d. hábito fértil; e. detalhe da flor; f-g. Tillandsia paraensis - f. hábito fértil; g. detalhe da flor; h. Tillandsia tenuifolia - detalhe da inflorescência; i. Tillandsia streptocarpa - detalhe da flor.

Figure 4 - a. Tillandsia bulbosa - detail of inflorescence; b-c. Tillandsia fasciculate - b. fertile habit; c. flower; d-e. Tillandsia monadelpha - d. fertile habit; e. detail of flower; f-g. Tillandsia paraensis - f. fertile habit; g. detail of flower; h. Tillandsia tenuifolia - detail of inflorescence; i. Tillandsia streptocarpa - detail of flower. 


\section{Agradecimentos}

As autoras agradecem à Norte Energia S.A., o acesso e a disponibilidade dos dados das espécies obtidos do Projeto Salvamento e Aproveitamento Científico da Flora da UHE Belo Monte; à Biota Projetos e Consultoria Ambiental Ltda., o apoio logístico durante a coleta de dados e a confecção do mapa; ao CNPq, a concessão da bolsa de doutorado da primeira autora; à CAPES, a concessão da bolsa de doutorado da segunda autora; aos curadores dos herbários Regina Célia Viana Martins (IAN), Rafaela Campostrini Forzza (RB) e Pedro Lage Viana (MG), por permitirem o acesso às coleções científicas; ao Engenheiro Florestal Willian de Lemos Guimarães, a confecção do mapa da área de estudo; e aos revisores anônimos.

\section{Referências}

Brummitt, R.K. \& Powell, C.E. 1992. Authors of plants names. Royal Botanic Gardens, Kew. 732p.

Dubs, B. 1998. Prodromus Florae Matogrossensis. Part I: Checklist of Angiosperms. Betrona, Verlaga. 444p.

Fidalgo, O.\& Bononi, V.L.R. 1984. Técnicas de coleta, preservação e herborização do material botânico. Instituto de Botânica (Manual, n. 4), São Paulo. 62p.

Forzza, R.C.; Costa, A.F.; Leme, E.M.C.; Versieux, L.M.; Wanderley, M.G.L.; Louzada, R.B.; Monteiro, R.F.; Judice, D.M.; Fernandez, E.P.; Borges, R.A.X.; Penedo, T.S.A.; Monteiro, N.P. \& Moraes, M.A. 2013. Bromeliaceae. In: Martinelli, G. \& Moraes, M.A. Livro vermelho da flora do Brasil. Andrea Jakobsson \& Instituto de Pesquisas do Jardim Botânico do Rio de Janeiro, Rio de Janeiro. Pp. 315-397.

Forzza, R.C.; Costa, A.; Siqueira Filho, J.A.; Martinelli, G.; Monteiro, R.F.; Santos-Silva, F.; Saraiva, D.P.; Paixão-Souza, B.; Louzada, R.B. \& Versieux, L. 2014. Bromeliaceae. In: Lista de espécies da flora do Brasil. Jardim Botânico do Rio de Janeiro. Disponível em <http://reflora.jbrj.gov/jabot/ floradobrasil/FB66>. Acesso em 15 julho 2014.

Givnish, T.J.; Millam, K.C.; Evans, T. M; Hall, J.C.; Pires, J.C.; Berry P.E. \& Sytsma, K.J. 2004. Ancient vicariance or recent long-distance dispersal? Inferences about phylogeny and South American - African disjunction in Rapateaceae and Bromeliaceae based on ndhF sequence data. International Journal of Plant Sciences 165: 35-54.

Givnish, T.J.; Millan, K.C.; Berry, P.E. \& Sytsma K.J. 2007. Phylogeny, adaptive radiation, and historical biogeography of Bromeliaceae inferred from ndhF sequence data. In: Columbus, J.T.; Friar, E.A.; Porter, J.M.; Prince, L.M. \& Simpson, M.G. Monocots: Comparative biology and evolution - Poales, Rancho Santa Ana Botanic Garden, Claremont. Pp. 3-26.
Givnish, T.J.; Barffus, M.H.J.; Van E.B.; Riina, R.; Schulte, K.; Horres, R.; Gonsiska, P.A.; Jabaily, R.S.; Crayn, D.M.; Smith, J.A.C.; Inverno, K.; Brown, G.K.; Evans, T.M.; Holst, B.K.; Luther, H.; Till, W.; Zizka, G.; Barry, P.E. \& Sytsma K.J. 2011. Phylogeny, adaptative radiation, and historical biogeography in Bromeliaceae: insights from an eight-locus plastid phylogeny. American Journal of Botany 98: 872-895.

Luther, H.E. 2012. An alphabetical list of bromeliad binomials. Marie Selby Botanical Gardens and Bromeliad Society International, Sarasota. 44p.

IBAMA. 2014. Lista das espécies da flora brasileira ameaçadas de extinção no Pará e demais estados do Bioma Amazônia. Disponível em <http://www. ibama.gov.br/documentos.flora-ameaçada $>$. Acesso em 5 agosto 2014.

IUCN. 2014. Red list of threatened species. Disponível em $<$ http://www.iucnredlist.org/>. Acesso em 5 agosto 2014 .

Koch, A.K.; Santos, J.U.M. \& Ilkiu-Borges, A.L. 2013. Bromeliaceae epífitas de uma área de conservação da Amazônia brasileira. Rodriguésia 64: 419-425.

Martinelli, G.; Vieira, C.M.; Gonzalez, M.; Leitman, P.; Piratininga, A.; Costa, A.F. da \& Forzza, R.C. 2008. Bromeliaceae da Mata Atlântica: lista de espécies, distribuição e conservação. Rodriguésia 59: 209-258.

Martinelli, G. \& Moraes, M.A. 2013. Livro vermelho da flora do Brasil. Andrea Jakobsson \& Instituto de Pesquisas do Jardim Botânico do Rio de Janeiro, Rio de Janeiro. Pp. 315-397.

Mello, B.M.; Abreu, L.L.; Koch, A.K.; Cardoso, A.L.R. \& Ilkiu-Borges, A.L. 2012. Bromeliaceae on the great curve of the Xingu River, Pará, Brazil. Rapid color guide 458, versão 1. Environnmental \& conservation programs. The Field Museum, Chicago. 2p. Disponível em <http://fm2.fieldmuseum.org/ plantguides/guideimages.asp? $\mathrm{ID}=533>$. Acesso $\mathrm{em}$ 15 julho 2014.

Mez, C. 1891. Bromeliaceae. In: Martius, C.F.P.; Eichler, A.G.; Urban, I. (eds.), Flora Brasiliensis. München, Wien, Leipzig. Vol 3, pars 3. Pp. 173-634.

MPEG - Museu Paraense Emílio Goeldi. 2008. Descrição e análise da flora da região do médio-baixo rio Xingú. Convênio MCT/MPEG/Camargo Corrêa/ Odebrecht/Andrade Gutierrez/ Eletrobrás/Fidesa. Relatório Técnico, Belém. 384p.

Nogueira-Braga, M.M. 1977. Anatomia foliar de Bromeliaceae da campina. Acta Amazonica 7: 1-74.

Quaresma, A.C. \& Medeiros, T.D.S. 2009. As bromélias. In: Jardim, M.A.G. Diversidade biológica das áreas de proteção ambiental Ilhas do Combu e AlgodoalMaiandeua, Pará, Brasil. Museu Paraense Emilio Goeldi, Belém. Pp. 71-78.

Quaresma, A.C. \& Jardim, M.A.G. 2012. Diversidade de bromeliáceas epífitas na Área de Proteção 
Ambiental Ilha do Combu, Belém, Pará, Brasil. Acta Botanica Brasilica 26: 290-294.

Quaresma, A.C. \& Jardim, M.A.G. 2013. Fitossociologia e distribuição espacial de Bromélias epifíticas em uma Floresta de Várzea Estuarina Amazônica. Revista Brasileira de Biociências 11: 1-6.

Ribeiro, J.E.S.; Hopkins, M.J.G.; Vicentini, A.; Sothers, C.A.; Costa, M.A.S.; Brito, J.M.; Souza, M.A.D.; Martins, L.H.P.; Lohmann, L.G.; Assunção, P.A.C.L.; Pereira, E.C.; Silva, C.F.; Mesquita, M.R. \& Procópio, L.C. 1999. Flora da Reserva Ducke: guia de identificação das plantas vasculares de uma floresta de terra-firme na Amazônia central. INPA, Manaus. 816p.

Salomão, R.P.; Vieira, I.C.G.; Suemitsu, C.; Rosa, N.A.; Almeida, S.S.; Amaral, D.D. \& Menezes, M.P.M. 2007. As florestas de Belo Monte na grande curva do rio Xingu, Amazonia Oriental. Boletim do Museu Paraense Emílio Goeldi, Ciências Naturais 2: $57-153$.

Scharf, U. \& Gouda, E.J. 2008. Bringing Bromeliaceae Back to Homeland Botany. Journal of the Bromeliad Society 58: 123-129.
SEMA. 2014. Espécies Ameaçadas. Disponível em $<$ http://www.sema.pa.gov.br/2009/03/27/9439>. Acesso em 5 agosto 2014.

Sousa G.M. \& Wanderley, M.G.L. 2007. Aechmea rodriguesiana (L.B.Sm.) L.B.Sm. (Bromeliaceae) uma espécie endêmica da Amazonia brasileira. Acta Amazonica 37: 517-520.

Sousa Junior, W.C.; Reid, J. \& Leitão, N.C.S. 2006. Custos e benefícios do complexo hidrelétrico Belo Monte: uma abordagem econômico-ambiental. Conservation Strategy Fund -CSF, Lagoa Santa. 90p.

Smith, L.B. \& Downs, R.J. 1974. Pitcairnioideae (Bromeliaceae). Flora Neotropica, Monograph 14: 1-662.

Smith, L.B. \& Downs, R. J. 1977. Tillandsioideae (Bromeliaceae). Flora Neotropica, Monograph 14: 663-1492.

Smith, L.B. \& Downs, R. J. 1979. Bromelioideae (Bromeliaceae). Flora Neotropica, Monograph 14: 1493-2141.

World Checklist of Selected Plant Families. Bromeliaceae. Disponível em $<$ http://apps.kew.org/ wcsp/incfamilies.do>. Acesso em 23 outubro 2014. 